assess the nature of such lesions using endoscopic ultrasound (EUS) prior to a biopsy.

Methods A total of 22 consecutive patients with portal hypertension who underwent an EUS evaluation between June 2008 and November 2011 for upper GI polypoid lesions found on endoscopy were included in the study. Procedure and pathology reports, obtained from patients' electronic records, were reviewed.

Results Of the 22 patients ( 16 men, 6 women, median age 66 ) who underwent upper GI endoscopy, 11 had lesions in the proximal stomach (gastro-oesophageal junction, fundus, gastric body) while eight had lesions in the distal stomach (antrum, pylorus) and three in the duodenum. Six (27.3\%) proved to be varices and $4(18.2 \%)$ polypoid lesions over varices ( 2 benign, 2 malignant). Whereas, 7 (31.8\%) patients had true polyps. The remaining lesions found on EUS included $1(4.5 \%)$ case of gastric fold, 1 gastric antral vascular ectasia (GAVE), one ulcer, 1 case of external compression and one patient had a normal EUS with no lesion seen. Of the 10 cases of varices and varices underlying polypoid lesions, 8 (80\%) were in the proximal stomach. Histology of non-vascular lesions under EUS were available in 11 patients, which showed 5 (45.5\%) inflammatory polyps, 2 (18.2\%) adenocarcinoma, 2 (18.2\%) adenoma, 1 $(9.1 \%)$ normal and one was reported as insufficient sample.

Conclusion Indeterminate upper GI lesions encountered during routine endoscopy in patients with portal hypertension are commonly either varices or may develop around varices. We recommend EUS evaluation prior to biopsying such lesions in order to avoid potential serious complications such as iatrogenic variceal bleed.

Competing interests None declared.

\section{PMO-195 IS THERE OPTIMUM PERIOD OF OBSERVATION POST DAYCASE ERCP? 12 MONTH EXPERIENCE IN A LARGE NON-TERTIARY CENTRE}

doi:10.1136/gutjnl-2012-302514b.195

${ }^{1} \mathrm{~A}$ Singhal, ${ }^{*} \mathrm{~A}$ Jayachandran, ${ }^{1} \mathrm{R}$ Faizallah. ${ }^{1}$ Gastroenterolgy, Wirral NHS Teaching Hospitals, Wirral, UK; ${ }^{2}$ Acute Medicine, Wirral NHS Teaching Hospitals, Wirral, UK

Introduction Daycase ERCP is practised in approximately 50\% centres in UK. Even in these centres there is no uniform policy for post ERCP observation or duration of hospital stay. We established daycase ERCP service in January 2010 on the Wirral, catering to 360000 population and hereby present our experience over a 12-month period.

Methods Data from Unisoft, GI Endoscopy reporting tool, was analysed to identify all the daycase ERCPs performed from 1 January to 31 December 2010. All the patients who for any reason stayed overnight after ERCP or re-attended hospital within 7 days, were identified from day ward registry and patient administrative system. Medical notes of all these patients were reviewed. All patients were closely monitored post ERCP in medical day ward for $4 \mathrm{~h}$ and were then allowed to eat and drink if there were no concerns. All patients were seen by the ERCPist prior to discharge. Results Total of 395 ERCPs were performed by three endoscopists in this period of which 195 (48\%) were as daycases. Difficulty level in all cases was Level 1-2 as per cotton et al. Indication of ERCP was pancreato biliary malignancy in 29 (15\%), stone disease in $160(82 \%)$ and previous bile leak $6(3 \%)$ patients. All procedures in our unit are done with therapeutic intent. 137 (72\%) patients underwent sphincterotomy and/or stent insertion. Previously placed stents were removed in the rest along with balloon trawl/stone extraction as needed. In all $32(16.4 \%)$ patients were admitted overnight. Of these, $13(6.6 \%)$ were elective admissions due to patient choice such as those who were elderly and lived alone. There were 7 (3.5\%) complications including 3 mild cholangits, 1 moderate cholangitis, 1 mild and 1 severe pancreatitis and 1 death as per accepted guidelines by Cotton et al. One patient who died, chose to stay back electively but died $12 \mathrm{~h}$ later with pulmonary embolism. Rest 13 (6.6\%) cases were advised to stay overnight because of suspected adverse event (commonest being post ERCP pain in 10 cases) but this was not substantiated on further investigations. Only one out of 195 patients $(0.5 \%)$, presented within 7 days with procedure related complication, namely mild cholangitis. Overall there were $8(4 \%)$ complications in 195 daycase ERCPs. Out of these 8, only $2(25 \%)$ presented within $0-2 \mathrm{~h}, 4(50 \%)$ in $2-6 \mathrm{~h}$ and rest $2(25 \%)$ after $12 \mathrm{~h}$ of the procedure.

Conclusion Daycase ERCP is a safe service. We propose that patients should be kept nil by mouth for $4 \mathrm{~h}$ post procedure and observed upto $6 \mathrm{~h}$ on the daycase unit. It is good practise for patients to be seen by the ERCPist prior to discharge. This would pick up majority of procedure related complications and enhances patient satisfaction.

Competing interests None declared.

\section{REFERENCE}

1. Williams E, et al. BSG audit of ERCP. Gut 2007;56:821-9.

\section{PM0-196 REDUCING TIME TO GASTROSCOPY IN UPPER GASTROINTESTINAL BLEEDING}

doi:10.1136/gutjpl-2012-302514b.196

${ }^{1} \mathrm{M}$ T Wong, ${ }^{1} \mathrm{~A}$ Sundaralingam, ${ }^{*} \mathrm{~S}$ Bains, ${ }^{1} \mathrm{~K}$ Shaw, ${ }^{2} \mathrm{~S}$ Afghan, ${ }^{2} \mathrm{~S}$ A Filson, ${ }^{1}$ I Gooding. 'Colchester General Hospital, Colchester, UK; ${ }^{2}$ Barts and The London Medical School, London, UK

Introduction Approximately 300-350 patients present to Colchester General Hospital with an upper Gastrointestinal (GI) bleed per year. Guidelines advise endoscopy within $24 \mathrm{~h}$ of presentation. To improve our performance, we introduced a new system for prioritising these requests and monitored the results with repeated audits. Methods An audit of all upper GI bleed cases was conducted over the same 3-month period (March-May) in 2009, 2010 and 2011. For each case we obtained the times of admission, Oesophagogastroduodenoscopy (OGD) request, procedure and discharge. The discharge summary, and where necessary the notes, were consulted to separate cases admitted for bleeding from those where bleeding occurred after admission for another reason. The main theatre logs were consulted for numbers of emergency out-of-hours OGDs. In an effort to tackle to poor waiting times, the Early Morning Bleeder (EMB) system was introduced in July 2009. Two slots are allocated daily (excluding weekends) for gastroscopy for cases of upper GI bleeding at the beginning of the working day. Requests are collected in a box in the Medical Assessment Unit daily at 0730. The Rockall Score is used for prioritisation. These three audits thus compare the situation before and after introducing the EMB system.

Results

\begin{tabular}{lllll}
\hline & & $\mathbf{2 0 0 9}$ & $\mathbf{2 0 1 0}$ & $\mathbf{2 0 1 1}$ \\
\hline Total cases & & 72 & 80 & 85 \\
Bleeder admissions & & 54 & 59 & 53 \\
Wait from & 0 & $3.7 \%$ & $11.9 \%$ & $15.1 \%$ \\
admission to & 1 & $29.6 \%$ & $35.6 \%$ & $49.1 \%$ \\
OGD (days) & $\geq 2$ & $68.5 \%$ & $52.5 \%$ & $35.8 \%$ \\
Mean wait for OGD (days) & 3.26 & $1.95^{*}$ & $1.66^{*}$ \\
Median length of stay (days) & 6 & 4 & $3^{*}$ \\
\hline
\end{tabular}

${ }^{*} \mathrm{p}<0.05$ compared to 2009.

Waits were significantly longer for cases admitted on Friday or Saturday.

Numbers of out of hours OGDs for bleeding were 12 (2007) and 11 (2008) before the EMB and 7 (2010) and 6 (2011) afterwards.

Conclusion The EMB system has reduced waits from presentation to OGD and length of hospital stay for patients presenting with upper GI bleeds. Patients are probably safer as the number of out of hours OGDs has fallen. There are plans to extend the service to include 
weekends. Hospitals with no current method for prioritising OGDs for bleeding should consider using this system.

\section{Competing interests None declared.}

\section{PMO-197 ENDOSCOPIC FINDINGS IN COLLAGENOUS COLITIS; NOT ALWAYS MICROSCOPIC}

doi:10.1136/gutjnl-2012-302514b.197

${ }^{1} \mathrm{~A}$ Smirnidis, ${ }^{1} \mathrm{~K}$ C Trimble, ${ }^{2} \mathrm{~A}$ Lessells, ${ }^{1} \mathrm{~A}$ Koulaouzidis. ${ }^{* 1}$ Centre for Liver \& Digestive Disorders, Royal Infirmary of Edinburgh, Edinburgh, UK; ${ }^{2}$ Pathology Department, Western General Hospital, Edinburgh, UK

Introduction CC is classically associated with normal or unremarkable colonoscopy. In the last few years, reports have been published revealing findings that are thought to be characteristic or even pathognomonic of CC, such as alteration of the vascular mucosal pattern, mucosal nodularity and a sequence of mucosal changes from defects/lacerations to cicatricial lesions. The aim of this study was to evaluate the frequency and type of endoscopic findings in patients diagnosed with CC in our centre.

Methods Setting: Tertiary hospital, outpatients. Design: Retrospective study. The database of Pathology Department was searched for patients who have been diagnosed with CC between May 2008 and August 2011. Endoscopy reports and endoscopic images were retrieved and reviewed.

Results 155 patients were diagnosed with CC in the study period. The indications for colonoscopy were altered bowel habit (acute or chronic diarrhoea) in 126/133; 33 patients reported associated weight loss. The reports from 123 patients (96F/27M; median age 68.7 yrs, range 37-91 yrs) were eventually retrieved and further reviewed. The colonoscopies had been carried out by consultant (medical/surgeons): 47\%, nurse endoscopist: 20\%, associate specialist: $13 \%$ and fellow or registrar: $10 \%$. Of the above cohort, 67 (54.4\%) patients had no endoscopic findings and 44 (35.7\%) had irrelevant to CC findings such as diverticulosis, polyps or telangiectasias. Twelve ( $n=12 ; 9.75 \%$ ) had findings previously described as consistent with CC. In particular: 7 (5.7\%) had mucosal erythema or oedema (patchy, mild granularity or congestion), 4 $(3.25 \%)$ had lacerations (cat-scratch mucosa or bigger mucosal breaks) and 1 of them had a few mucosal scars. The sigmoid and the descending colon were the main colonic parts affected (in $7 / 12$ cases) and the rest were found in the caecum-ascending colon area (4/12) while there was only one patient in which the entire large bowel was affected.

Conclusion A significant minority of patients with CC (almost 10\%) presented endoscopic findings indicative of CC. Furthermore, $4 \%$ had findings that are considered pathognomonic for CC. Although still the subject of isolated cases reports, the endoscopic appearances of CC are becoming more familiar among the endoscopic community.

Competing interests None declared.

\section{REFERENCE}

1. Distinct colonoscopy findings of microscopic colitis: Not so microscopic after all? World J Gastroenterol 2011;17:4157-65.

\section{PMO-198 POST-OPERATIVE ENDOSCOPY IN BARIATRIC SURGERY PATIENTS}

doi:10.1136/gutjnl-2012-302514b.198

${ }^{1} \mathrm{~A}$ Desai, ${ }^{*}{ }^{1} \mathrm{G}$ Bahra, ${ }^{2} \mathrm{~A}$ Thillainayagam, ${ }^{3} \mathrm{~A}$ Ahmed. ${ }^{1}$ Foundation Year Doctor, Charing Cross Hospital, London, UK; ${ }^{2}$ Gastroenterology, Charing Cross Hospital, London, UK; ${ }^{3}$ Bariatric Surgery, Charing Cross Hospital, London, UK

Introduction Bariatric surgical patients may require endoscopy in the post-operative phase. The current study analyses the indications and findings of upper GI endoscopy (OGD) in post-operative bariatric surgery patients.

Methods A retrospective analysis of all bariatric surgery patients referred for oesophagogastroduodenoscopies (OGDs) at Charing Cross Hospital from 1 January 2009 to 30 October 2011. The Endoscopy units' electronic database of OGDs performed was analysed to determine how many bariatric surgery patients had OGDs postoperatively. Further sub-analysis was done for each operation type.

Results During this time period 1093 bariatric surgeries were performed. These included 542 laparoscopic gastric bypasses, 220 laparoscopic gastric bands, 223 laparoscopic sleeve gastrectomies and 108 revisional bariatric procedures. 147 OGDs were performed on a total of 116 Bariatric surgical patients, with 23 patients having had more than one OGD. Of these 147, 103 were done post-operatively; $58(56.3 \%)$ post-roux-en-y gastric bypass, 34 (33\%) post-gastric band insertion, $6(5.8 \%)$ post-gastric sleeve gastrectomy and $5(4.9 \%)$ post- bariatric revisional surgery. Indications for OGDs were abdominal pain $(44.7 \%)$, vomiting (15.5\%), haematemesis/malaena (9.7\%), failure of weight loss (7.8\%), follow-up for previous scopes/ imaging $(6.8 \%)$, reflux symptoms $(3.9 \%)$, dysphagia $(3.9 \%)$, interventional purposes ( 3 naso-jejunal tube insertions and 1 stricture dilatation) (3.9\%), suspected abnormal positioning/band erosion $(2.9 \%)$ and microcytic anaemia (0.9\%). Of the 34 gastric band OGDs done 13 were normal and 21 showed abnormalities including 7 gastric band erosions, 6 with mucosal inflammation, 2 with insufficient band compression, 2 with abnormal band position and 2 hiatal hernias. Of the 58 post-bypass endoscopies done 33 were normal, 15 showed anastomotic/pouch ulceration/inflammation/ erosion, 4 showed signs of recent haemorrhage and 3 oesophageal irritation. Out of 6 post-sleeve OGDs 2 were normal, 1 was done for an interventional stricture dilatation, 1 showed a gastric stricture, 1 oesophageal candidiasis and 1 a hiatal hernia. Four OGDs out of 5 done post-revisional surgery were normal. Of all OGDs referred for post-operative abdominal pain, $50 \%$ yielded abnormal findings.

Conclusion Endoscopy units need to be familiar with and prepared for bariatric surgery patients as post-operatively a substantial number will need endoscopic postoperative assessments. In our study $9.4 \%$ of all postoperative bariatric surgery patients underwent endoscopy, the commonest referral reason was abdominal pain and the commonest finding was normal.

Competing interests None declared.

\section{PMO-199 COMPARATIVE STUDY OF SAMPLE ADEOUACY OF 25G VS 22G NEEDLE IN ENDOSCOPIC ULTRASOUND (EUS) GUIDED FINE NEEDLE ASPIRATE (FNA) OF SOLID LESIONS}

doi:10.1136/gutjnl-2012-302514b.199

${ }^{1} \mathrm{~A}$ U Murugananthan, ${ }^{*}{ }^{1} \mathrm{~T}$ Hong, ${ }^{1,2} \mathrm{R}$ Y Chen. ${ }^{1}$ Gastroenterology, St Vincent's Hospital, Melbourne, Australia; ${ }^{2}$ Western Health, St Vincent's Hospital, Melbourne, Australia

Introduction Optimal needle size in achieving greatest diagnostic yield from EUS- guided FNA remains unclear.

Aim We prospectively compared sample adequacy and safety of FNA of solid lesions between 25G and 22G ( $\mathrm{Cook}^{\mathrm{TM}}$ ) needle at two tertiary centres.

Methods Prospective data from two sites was collected between November 2008 and November 2011. A single operator alternated on a case-by-case basis between a 25G and 22G needle. A cytopathologist was present to assess adequacy of sample. The operator could switch needle size if required.

Results 152 patients undergoing 165 FNA were analysed $(42 \mathrm{M} / 30 \mathrm{~F}$ mean age 59). 76 patients had FNA with a $22 \mathrm{~F}$ needle and 76 with 